\title{
Discussion of the experience and improvement of an enhanced recovery after surgery procedure for minimally invasive lobectomy: a cohort study
}

\author{
Haoxiang $\mathrm{Ni}^{1} \wedge$, Peng $\mathrm{Li}^{2}$, Zhe Meng ${ }^{2}$, Tianwei Huang ${ }^{2}$, Liang $\mathrm{Shi}^{2}$, Bin $\mathrm{Ni}^{2}$ \\ ${ }^{1}$ The Second Clinical Medical College of Xuzhou Medical University, Xuzhou, China; ${ }^{2}$ Department of Thoracic Surgery, The First Affiliated \\ Hospital of Soochow University, Suzhou, China \\ Contributions: (I) Conception and design: B Ni; (II) Administrative support: H Ni; (III) Provision of study materials or patients: P Li, Z Meng; (IV) \\ Collection and assembly of data: H Ni, P Li, Z Meng, T Huang, L Shi; (V) Data analysis and interpretation: H Ni, P Li; (VI) Manuscript writing: All \\ authors; (VII) Final approval of manuscript: All authors. \\ Correspondence to: Bin Ni. Department of Thoracic Surgery, The First Affiliated Hospital of Soochow University, 188 Shizi Street, Gusu District, \\ Suzhou 215006, China. Email: nb_fyywk@163.com.
}

Background: It is of great significance to explore a path for expedited recovery from thoracic surgery for patients undergoing minimally invasive lobectomy to ensure their rapid and smooth recovery and to conserve medical resources.

Methods: We analyzed 629 cases from the Department of Thoracic Surgery, First Affiliated Hospital of Soochow University from January 2018 to January 2020. According to the length of postoperative stay (LOS) and perioperative management, the 629 patients were divided into group A [routine management group (RMG)], group B [rapidly recovery group (RRG), LOS $>72 \mathrm{~h}$ ], and group C (RRG, LOS $\leq 72 \mathrm{~h}$ ). The $t$-test and chi-square test were used to compare the postoperative complications (PC), chest tube indwelling time (CTIT), LOS, postoperative opioid dosages (POD), and total costs (TC) of the 3 groups.

Results: Compared with the RMG, the LOS, PC, CTIT, POD, and TC of the RRG were statistically significantly ameliorated $(\mathrm{P}<0.05)$. When compared with group $\mathrm{A}$, the $\mathrm{PC}(18.9 \%$ vs. $38.8 \%)$, LOS $(2.74 \pm 0.80$ vs. $5.70 \pm 1.10 \mathrm{~d})$, CTIT $(46.1 \pm 18.5$ vs. $123.6 \pm 34.8 \mathrm{~h})$, and TC $(¥ 51,517 \pm 7,217$ vs. $¥ 65,781 \pm 8,200)$ of group C were all decreased. Compared with group A, group B had more preoperative complications, less CTIT, but no significant difference in LOS, PC, and TC during hospital stay. Compared with group B, group C had less preoperative complications, and reduced LOS, CTIT, and TC. The POD in group A was significantly higher than that in groups B and C.

Conclusions: Use of an enhanced recover after surgery (ERAS) protocol can reduce the postoperative complications and shorten the LOS. Some high-risk patients cannot be discharged within $72 \mathrm{~h}$ after surgery, but their incidence of grade II postoperative lung complications can be decreased and they can benefit from ERAS. Single intercostal nerve block, COX-2 selective inhibitor, and removal of the chest tube as early as possible can improve the ERAS.

Keywords: Enhanced recover after surgery (ERAS); video-assisted thoracic surgery (VATS); minimally invasive lobectomy

Submitted Oct 29, 2021. Accepted for publication Dec 22, 2021.

doi: 10.21037/atm-21-6493

View this article at: https://dx.doi.org/10.21037/atm-21-6493

$\wedge$ ORCID: 0000-0002-5989-9757. 


\section{Introduction}

Lung cancer is the most threatening cancer to human health. According to the World Health Organization International Agency for Research on Cancer (IARC), in 2012, there were an estimated 1.825 million cases of lung cancer and 1.590 million deaths worldwide, ranking it the first in morbidity and mortality of malignant tumors worldwide (1) and in China (2,3). In recent years, with the popularization of low-dose spiral computed tomography (CT), the early detection rate of lung cancer is increasing, along with the number of people requiring treatment. The large number of patients has significantly increased the pressure on clinical medical resources. Therefore, it is of great significance to explore a rapid recovery path suitable for the proliferation of thoracic surgery in China and for patients undergoing minimally invasive lobectomy to ensure the rapid and smooth recovery of patients and to preserve medical resources.

Enhanced recover after surgery (ERAS) was first reported by the Danish doctor Kehlet in 2001. It refers to the adoption of a series of optimized measures of perioperative management supported by evidence-based medicine, so as to reduce the physiological and psychological posttraumatic stress response of patients caused by the operation and achieve the purpose of accelerating their recovery (4). In recent years, the scope of ERAS protocols has gradually expanded, starting from the regulation of preoperative homeostasis, selection of perioperative anesthesia and analgesia programs, postoperative functional training, and so forth, until a complete set of measures was gradually formed, and then gradually extended to gastric cancer, breast cancer, and thoracic surgery (5-8). At present, ERAS has been studied and reported on in various surgical fields, and may become the ethos of perioperative management of surgery in the future (9-12).

In recent years, with the development of minimally invasive thoracic surgery, patients have experienced many benefits, such as shorter surgery time, decreased incidence of postoperative complications, and shortened postoperative hospital stay (13). These results are consistent with the purpose of ERAS. For many thoracic surgeons, there is no contradiction between minimally invasive technology and ERAS protocols, and they are mutually complementary, thus bringing greater benefits to patients (14). In 2018, the European Thoracic Society (ETS) proposed guidelines on rapid recovery of thoracic surgery, which discussed management strategies and evidence levels of rapid recovery in the perioperative period from the perspective of evidence-based medicine, and unified some thoughts to a certain extent (15).

In this context, we have carried out research and practice of ERAS protocols in thoracic surgery. Under the premise of safety, through continuous optimization of the evidence-based medicine measures, our research has aimed to help reduce patients' length of recovery time as much as possible. Continuous clinical practice has helped us to refine the advancements in minimally invasive surgery analgesia and multimodal analgesia, precise analgesia, chest tube management, ensure long-term lung function recovery, and so on. Many aspects still have a lot of room for improvement, including optimization of the selection process for therapeutic measures to more effectively implement rapid rehabilitation management, how to maximize the meeting of clinical needs, and preparing the protocol for clinical promotion.

Our primary objectives were as follows: (I) validate the feasibility and necessity for enhanced ERAS management in the perioperative management of minimally invasive lobectomy; and (II) optimize the perioperative management regulations of ERAS in thoracic surgery to make it more suitable for clinical promotion.

We present the following article in accordance with the STROBE reporting checklist (available at https://dx.doi. org/10.21037/atm-21-6493).

\section{Methods}

\section{Research design}

This study was based on cohort and retrospective studies. All procedures performed in this study involving human participants were in accordance with the Declaration of Helsinki (as revised in 2013). Individual consent for this retrospective analysis was waived. The ethical approval was not required. Because ERAS protocol has been in normal process in thoracic surgery of our hospital, this paper reviews the previous cases, mainly to summarize and improve the experience and of ERAS protocol and prove the effectiveness of rapid rehabilitation. An analysis was performed on 629 patients that underwent thoracoscopic lobectomy in the Department of Thoracic Surgery, the First Affiliated Hospital of Soochow University from January 2018 to January 2020. All participants were derived from the same surgical and care team. The exclusion criteria were as follows: (I) secondary surgery due to complications; (II) lack 
of perioperative related clinical data; (III) the formulated perioperative rehabilitation management regulations were not strictly followed during the perioperative period; (IV) patients who had refused early discharge. All criteria were designed to reduce possible information bias with selection bias. The research design is displayed in Figure 1.

In the perioperative period, group A was defined as the routine management group (RMG), and those receiving ERAS protocols management were classified as the rapid recovery group (RRG). In the RRG, a postoperative hospitalization time $>72 \mathrm{~h}$ was classified as the nonearly discharge group (group B), and a postoperative hospitalization time $\leq 72 \mathrm{~h}$ was classified as the early discharge group (group C). Relevant clinical data were collected, including preoperative general condition, postoperative complications (PC), chest tube indwelling time (CTIT), length of postoperative stay (LOS), postoperative opioid dosages (POD), and total costs (TC).

\section{Research methods}

Patients undergoing rapid perioperative rehabilitation management (group B and group C) began the process immediately after admission. Patients undergoing conventional perioperative management (group A) were managed according to the routine perioperative plan. The incidence rate of PC, CTIT, pain score of the first 3 days after the operation, POD for the first 3 postoperative days, total number of steps walked after the operation, total number of steps walked in the $24 \mathrm{~h}$ of the first day after surgery, total length of stay after surgery, and total cost of hospital stay were analyzed. The visual analogue scale (VAS) was used to score pain and nausea. The CTIT was calculated in hours from the end of surgery to the time of extubation. Postoperative hospital stay was calculated in days from the end of surgery to the time of discharge. The number of steps walked on the postoperative day was the number of active steps between the end of surgery and 8:00 am on the first day after surgery, and was measured by wrist pedometer. The number of steps walked on the first day after surgery was the number of active steps from 8:00 am on the first day after surgery to 8:00 am on the second day after surgery. The total cost of hospitalization was the total cost incurred during hospitalization.

\section{Perioperative routine management and rapid rehabilitation management}

Patients in the RMG were managed in the perioperative period according to the current management process widely accepted in the thoracic surgery ward, while patients in the RRG were managed according to the design of our improved rapid recovery (ERAS) process.

(I) In preoperative education, RRG focused on adequate surgical education, the trained nurse taught the patients about the rapid rehabilitation concept, and the significance of various preoperative and postoperative treatment measures, thus promoting patients to cooperate. The details of each process and matters needing attention were introduced, with emphasis on preoperative liquid food, $2 \mathrm{~h}$ early postoperative ambulation, bed cough and bedside urinating, learning how to complete the VAS pain score, and so on.

(II) In terms of preoperative evaluation, the RMG underwent disease diagnosis and surgical method evaluation, anesthesia and surgical risk evaluation for those with pulmonary nodules according to the current medical routine; the RRG were also required to undergo risk assessment of venous thromboembolism (Caprini score scale) (16) and nutritional status assessment (nutrition risk screening form NRS-2002 table).

(III) In terms of nutrition management, the RMG was subject to habitual preoperative fasting for $12 \mathrm{~h}$ and the prohibition of drinking for $6 \mathrm{~h}$. The RRG switched from a solid diet to fasting for $6 \mathrm{~h}$ before surgery, and drinking sugar water or $400 \mathrm{~mL}$ of sugar saline for $2 \mathrm{~h}$ before surgery, with the aim of investigating whether this could improve patients' tolerance to surgery and reduce interference of the internal environment during surgery. Patients with preoperative nutritional status of NRS-2002 $\geq 3$ points were given enteral and parenteral nutritional support therapy before surgery.

(IV) In terms of surgical management, the 2 groups of patients adopted the same surgical method, all surgical operations were completed in the form of thoracoscopic single-operation hole. During the operation, the measures for maintaining hemostasis and prevention of lung leakage were strengthened. Exact hemostasis was performed at the hilum of lung and the mediastinal lymph node. Hemostatic materials were available for use to prevent the occurrence of chylothorax. There was no specific difference between the 2 groups in terms of surgical methods. In the RRG, intercostal nerve block was 


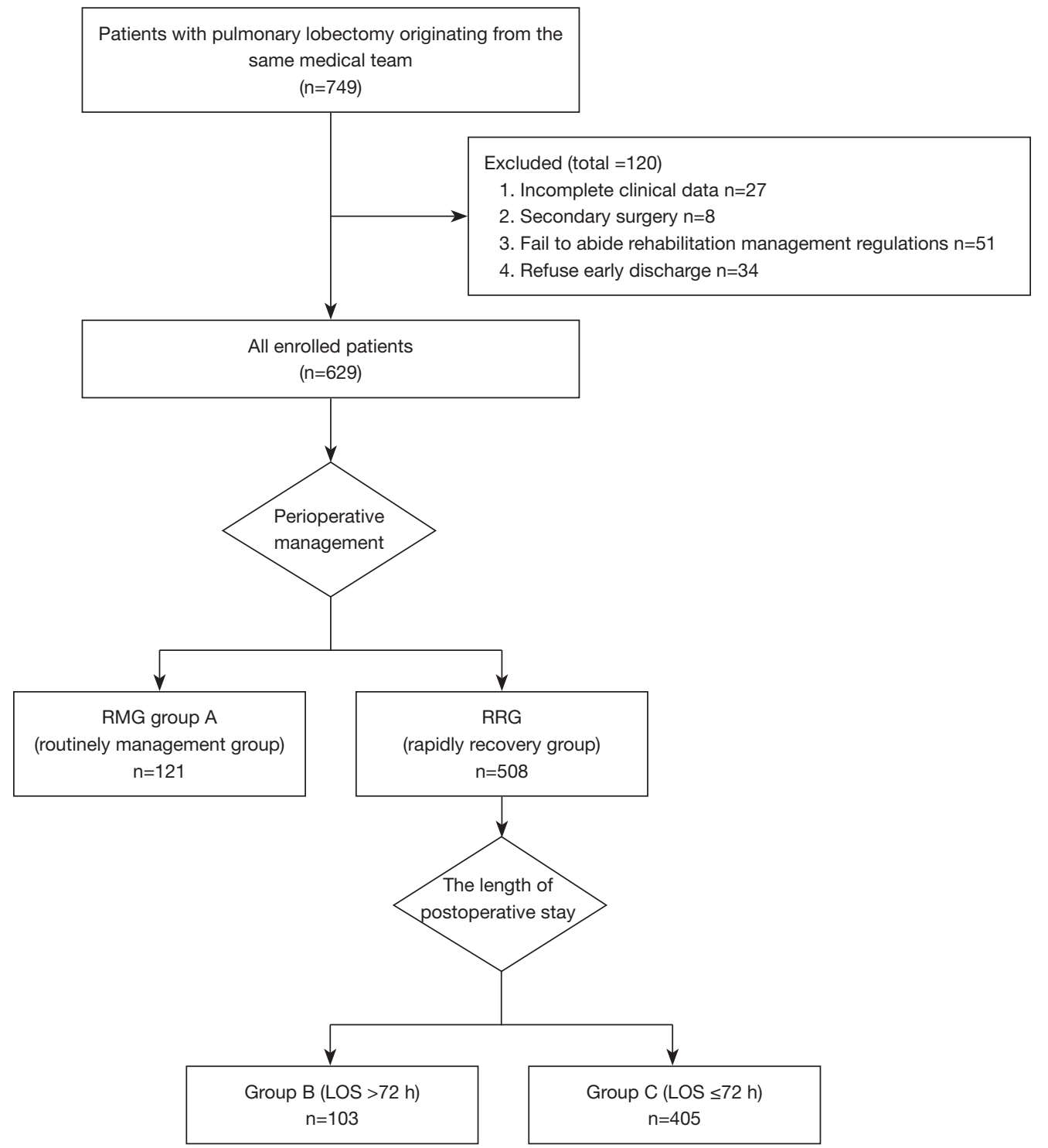

Figure 1 Research design. RMG, routine management group; RRG, rapid recovery group; LOS, length of postoperative stay.

performed under thoracoscopic vision from the upper operation hole to the lower observation hole in the chest closure during the operation, and in the RRG, thermal insulation treatment was closely observed during the operation.

(V) In terms of pain management, the RMG adopted the traditional postoperative pain management method. When the postoperative VAS score of patients was greater than or equal to 4 , opioid analgesia was used. In the RRG, pre-analgesia and multimode analgesia (including intraoperative intercostal nerve block and postoperative intravenous or oral administration of selective COX-2 inhibitorbased analgesics) were administered by oral nonsteroidal anti-inflammatory drugs (NSAIDs) COX2 inhibitor 2 days before the operation, without intravenous analgesia pump, and opioids were avoided as much as possible.

(VI) In terms of chest tube management, the RMG adopted the traditional upper thoracic lobectomy to place one $28 \mathrm{~F}$ chest tube for exhaust and drainage, and the lower pulmonary lobectomy to place 1 or 2 chest tubes under the condition of apparent air leakage. The standard for chest tube removal 
was chest drainage flow less than $200 \mathrm{~mL} / \mathrm{d}$ after surgery. In the RRG, $28 \mathrm{~F}$ thoracic tubes were placed at the top of the chest for drainage, and the standard for removal of the thoracic drainage tube was modified to be non-blood septic chyle of the thoracic drainage fluid, less than $500 \mathrm{~mL} / \mathrm{d}$, with no air leakage and lung reconstruction on the chest film.

(VII) In terms of the management of bleeding and thrombus, the 2 groups were all assessed according to the preoperative risk of venous thromboembolism (VTE), we use the Caprini Score to assess the risk of perioperative venous thrombosis, and no additional measures were taken for patients with mild risk. The patients with moderate risk were treated with thromboembolism; those with severe risk were treated with low-molecular-weight heparin after surgery; and those with severe risk were treated with oral anticoagulant drugs, such as aspirin, when the bleeding risk was stopped.

(VIII) In terms of catheter management, the RMG had the catheter placed before surgery after anesthesia and it was removed $48-72 \mathrm{~h}$ after surgery. In the RRG, urinary catheters were not indwelling before surgery. In a few patients whose urine volume needed to be observed during anesthesia, urinary catheters were indwelling before surgery after anesthesia and were removed before the patients woke up after surgery.

(VIIII) In terms of circulation management, the RMG, according to usual practice, had an intraoperative fluid supply of $1,000 \mathrm{~mL}$ and a postoperative fluid supply of $1,500 \mathrm{~mL} / \mathrm{d}$. In the $\mathrm{RRG}$, the intraoperative limited intravenous fluid supply was about $500 \mathrm{~mL}$, and the daily postoperative fluid supply was less than $1,000 \mathrm{~mL}$.

(X) In terms of postoperative management, the RMG did not emphasize early postoperative discontinuation and activity, and did not have specific activity requirements for. The patients in the RRG were required to get out of bed and go to the toilet on the day after surgery. They were required to get out of bed with chest tube at least twice on the first day after surgery, and walk for at least $6 \mathrm{~min} / 200 \mathrm{~m}$ each time. The total amount of walking was at least 2,000 steps within $24 \mathrm{~h}$. All the patients in the RRG received respiratory trainers after surgery (Table 1).

\section{Extubation criteria, pain score, discharge, and readmission criteria}

The extubation standard was as follows: the patient's vital signs were stable, without obvious palpitations, shortness of breath, and other discomfort; chest radiograph showed no obvious accumulation of fluid and gas in the chest cavity. For the RMG, $24 \mathrm{~h}$ drainage fluid was less than $200 \mathrm{~mL} / \mathrm{d}$, and $24 \mathrm{~h}$ drainage fluid in the RRG was less than $500 \mathrm{~mL} / \mathrm{d}$, and the drainage fluid was non-purulent, bloody, and chylous.

Pain score: all participants were assessed without opioid analgesics, and each individual was assessed once in the morning and again in the evening. When the pain score was $\geq 4,1 \mathrm{mg} / \mathrm{kg}$ of pethidine hydrochloride was used for intramuscular analgesia; 0 score: no pain of activity and forced cough;1 scores: slight pain in turning over and coughing; 2 scores: quiet and without pain when supine, but when turning over or coughing there was pain, breathing out was ok, but there was fear of coughing because of the pain; 3 scores: quiet while prostrate without pain, but pain was obvious when deep breathing or rolling cough, slightly because of the pain and fear of deep breathing and cough; 4 scores: occasional mild pain during silence, fear of deep breathing and coughing due to the pain, but still effective cough; 5 scores: often having mild pain in the supine position, obvious fear of cough caused by the pain, basically ineffective cough; 6 scores: obvious pain even while resting; 7 scores: frequent obvious pain while resting; 8 scores: pain while resting, sweating due to pain; 9 scores: feeling dead due to postoperative pain; 10 scores: unable to stand the pain.

The discharge criteria were as follows: (I) the patient was generally in good condition, with no symptoms such as chest pain, chest tightness, palpitation, and shortness of breath; (II) the chest tube had been removed or retained in situ for $\geq 6$ days, the remaining lung retentions were good, the drainage fluid was without pus, blood, and chylous, only a few bubbles during coughing which can be discharged with the chest tube; (III) the wound had recovered well without any infection signs.

Postoperative recatheterization criteria were as follows: (I) the chest radiograph suggested that the effusion plane was as high as the 4th intercostal level, and the pleural color ultrasound suggested that the effusion depth was more than $70 \mathrm{~mm}$ (17); (II) bedside chest perforation or resituated closed thoracic drainage tube.

The readmission criteria were as follows: (I) severe pulmonary infection confirmed by clinical symptoms and 
Table 1 Comparison of Perioperative management between RMG and RRG

\begin{tabular}{|c|c|c|}
\hline Period & RMG & RRG \\
\hline Intraoperative & $\begin{array}{l}\text { (I) Preoperative prophylactic } \\
\text { administration of antibiotics; (II) } \\
\text { preoperative indwelling catheter and } \\
\text { return to ward; (III) minimally invasive } \\
\text { surgery; (IV) superior lobectomy was } \\
\text { performed with two roots, and middle } \\
\text { and lower lobectomy was performed } \\
\text { with a single } 28^{\#} \text { thoracic duct } \\
\text { drainage; }(\mathrm{V}) \text { intraoperative fluid supply } \\
\text { was } 1,000 \mathrm{~mL} \text {, and postoperative fluid } \\
\text { supply was } 1,500 \mathrm{~mL} / \mathrm{d}\end{array}$ & $\begin{array}{l}\text { (I) Preoperative prophylactic use of antibiotics; (II) protective lung ventilation } \\
\text { strategy and body temperature protection measures; (III) the catheter was } \\
\text { removed without indwelling or after surgery; (IV) endoscopic vision of the } \\
\text { lower intercostal nerve block; (V) carefully expand the lung to suture air } \\
\text { leakage and hemostasis before closing the chest cavity; (VI) all } 28^{\#} \text { chest } \\
\text { tubes were placed at the top of the chest for drainage; (VII) intraoperative } \\
\text { intravenous fluid supply was limited to about } 500 \mathrm{~mL} \text {, and postoperative daily } \\
\text { fluid supply was less than } 1,000 \mathrm{~mL}\end{array}$ \\
\hline Postoperative & $\begin{array}{l}\text { (I) On demand opioid intramuscular } \\
\text { analgesia; (II) prevention of excessive } \\
\text { vomiting; (III) the chest tube was } \\
\text { removed after <200 } \mathrm{mL} / \mathrm{d} \text {; (IV) do not } \\
\text { restrict intravenous fluid, according } \\
\text { to the patient's will to get out of bed, } \\
\text { strengthen cough to promote cough; }(\mathrm{V}) \\
\text { prevention of deep vein thrombosis; }(\mathrm{VI}) \\
\text { stepped-up routine back patting for } \\
\text { expectoration }\end{array}$ & $\begin{array}{l}\text { (I) Intravenous NSAIDs COX-2 inhibitors were used every } 12 \mathrm{~h} \text { for the first } \\
3 \text { days after surgery, and oral administration continued for } 4 \text { days on the } \\
\text { fourth day; (II) start to get out of bed } 6 \mathrm{~h} \text { after the operation, and set the } \\
\text { postoperative walking amount; (III) control intravenous fluids; (IV) prevent } \\
\text { nausea and vomiting; (V) early removal of chest tube }(<500 \mathrm{~mL} / \mathrm{d}) \text {; (VI) } \\
\text { prevention of deep vein thrombosis; (VII) get out of bed on the day after the } \\
\text { operation. On the first day after the operation, get out of bed with chest tube } \\
\text { twice a day, } 6 \mathrm{~min} / 200 \mathrm{~m} \text { or more each time; (VIII) use a breathing trainer }\end{array}$ \\
\hline
\end{tabular}

RMG, routine management group; RRG, rapid recovery group; NSAIDs, non-steroidal anti-inflammatory drugs.

chest CT requires intravenous anti-inflammatory treatment; (II) a large amount of pleural effusion or empyema requiring hospitalization for observation and treatment; (III) postoperative spontaneous pneumothorax requiring inpatient catheterization; (IV) other conditions requiring re-hospitalization.

\section{Part of the identification criteria for preoperative complications and postoperative complications}

\section{Low lung function}

Preoperative lung function of patients with the maximal voluntary ventilation (MVV) $<60 \%$, the first second forced expiratory volume (FEV1) /forced vital capacity (FVC) $<70 \%$ (18).

\section{Postoperative pulmonary infection}

Chest radiograph or chest CT showed pulmonary infiltrating and exudative patchy image and conformed to 2 of the following 3 conditions: (I) hyperpyrexia; (II) leukocyte
$>12 \times 10^{9} / \mathrm{L}$ or $<4 \times 10^{9} / \mathrm{L}$; (III) lung auscultation showed new or altered rales; (IV) positive sputum culture (19).

\section{Coronary artery disease}

Preoperative computed tomography angiography (CTA) or coronary angiography suggested the presence of more than 1 coronary artery stenosis $\geq 70 \%$, and preoperative anticoagulation treatment was required.

\section{Renal insufficiency}

Glomerular filtration rate (GFR) $\leq 29 \mathrm{~mL} /\left(\min \cdot 1.73 \mathrm{~m}^{2}\right)$ for more than 3 months or requiring renal dialysis.

\section{Intracranial vascular stenosis}

Preoperative head CTA or head transcranial Doppler ultrasonography (TCD) indicated intracranial blood vessels of at least level III narrowing (70\% or higher), requiring preoperative anticoagulants.

\section{Continuous air leakage}

Bubbles could be seen escaping from the chest drainage bottle due to cough or activity after surgery, and the 
Table 2 Grading standards of postoperative complication (23)

\begin{tabular}{ll}
\hline Grade & Description \\
\hline I & Without any intervention \\
IIIA & Only drug intervention \\
& $\begin{array}{l}\text { Need not under the general anesthesia further } \\
\text { IIIB }\end{array}$ \\
IVA & Need further intervention under general anesthesia \\
IVB & Need multiple organ support life \\
V & Death due to the complications
\end{tabular}

Level I pulmonary complications: prolonged gas leakage; Level II and above pulmonary complications: other postoperative pulmonary complications except prolonged air leakage, including pulmonary infection, atelectasis, acute lung injury, and asthma, are collectively referred to as level II and above pulmonary complications.

duration was $\geq 6$ days.

\section{Asthma}

Auscultation of wheezes or respiratory sounds in the lungs; the blood gas analysis indicated that the partial pressure of oxygen $\left(\mathrm{PaO}_{2}\right)$ decreased and the partial pressure of carbon dioxide $\left(\mathrm{PaCO}_{2}\right)$ increased. The symptoms were relieved after symptomatic treatment such as bronchiectasis, and the diagnosis was made after the diagnosis was differentiated from secretions blockage, allergic reaction, pulmonary edema, aspiration, pulmonary embolism, pneumothorax, and so on $(20,21)$.

\section{Nausea and vomiting}

The patient was evaluated when no antiemetic drug had been used and nausea and vomiting were present, and the vomiting visual simulation score was used as the basis for the determination of a single episode greater than 7 points. A score of 1-3 marks indicated mild nausea without vomiting, which did not interfere with oral feeding; a score of 4-6 indicated moderate nausea with vomiting, and drinking of water was still possible despite poor oral feeding; a score of 7-10 indicated severe nausea with obvious vomiting, and the patient was unable to take water through the mouth due to nausea or vomiting. The maximum value was 10 , indicating hyperemesis (22).

\section{Grading standards of postoperative complication (Table 2) (23)}

In order to better study the postoperative complications, the postoperative complications are classified according to whether drugs or surgical intervention and organ function support are needed, from grades I to V (Table 2).

\section{Intraoperative intercostal nerve block}

All the patients in the RRG underwent at least 6 intercostal nerve blocks around the upper intercostal space of the operation hole. Specific operation: locate the intercostal area, reveal the small head of the ribs horizontal, exposure the lower edge of the intercostal nerve root as the needle entry point, and conduct vertical puncture with a fine needle. After the extraction of no blood, inject about $2-3 \mathrm{~mL}$ anesthetic fluid, and fill the peripheral pleura of the intercostal nerve. Anesthetic solution ratio: $0.75 \%$ ropivacaine hydrochloride injection (AstraZeneca $\mathrm{AB}$, Södertälje, Sweden) $10 \mathrm{~mL}$, 2\% lidocaine hydrochloride injection $5 \mathrm{~mL}, 5 \mathrm{~mL}$ normal saline.

\section{Use of NSAIDs in perioperative period}

Patients in the RRG were given the NSAIDs COX-2 inhibitor Celecoxib Capsule (200 mg, Pfizer, Inc., New York, NY, USA) orally bid. from the second day before surgery. Intravenous NSAIDs COX-2 inhibitor was administered $\mathrm{Q} 12 \mathrm{H}$ for the first 3 days after the operation with Parichib Sodium for injection (Dynastat, $40 \mathrm{mg}$, Pfizer Inc.), followed by oral administration of Celecoxib Capsule on the fourth day (200 mg, bid).

\section{Statistical analysis}

The software GraphPad Prism 7.0 (GraphPad Software, La Jolla, CA, USA was used to process the data. In the RMG (group A) and the RRG (group B and group C), the general data and the research indicators were measured by $t$-test and chi-square test. A $\mathrm{P}$ value $<0.05$ indicated that the difference was significant. Cases with missing clinically relevant data were not included in this study.

\section{Results}

In this study, a total of 629 patients were enrolled. In addition to the number of chest tubes, for age, operation time, intraoperative blood loss, number of resected lymph nodes, surgical site, and pathological typing, the $\mathrm{P}$ value of $>0.05$ was not significantly different between the RMG and the RRG (Table 3).

Among the patients in the RMG, there were 36 cases of preoperative complications, accounting for $29.8 \%$. In the RRG, 161 patients had preoperative complications, 
Table 3 Comparison of general data of patients in the RMG and RRG

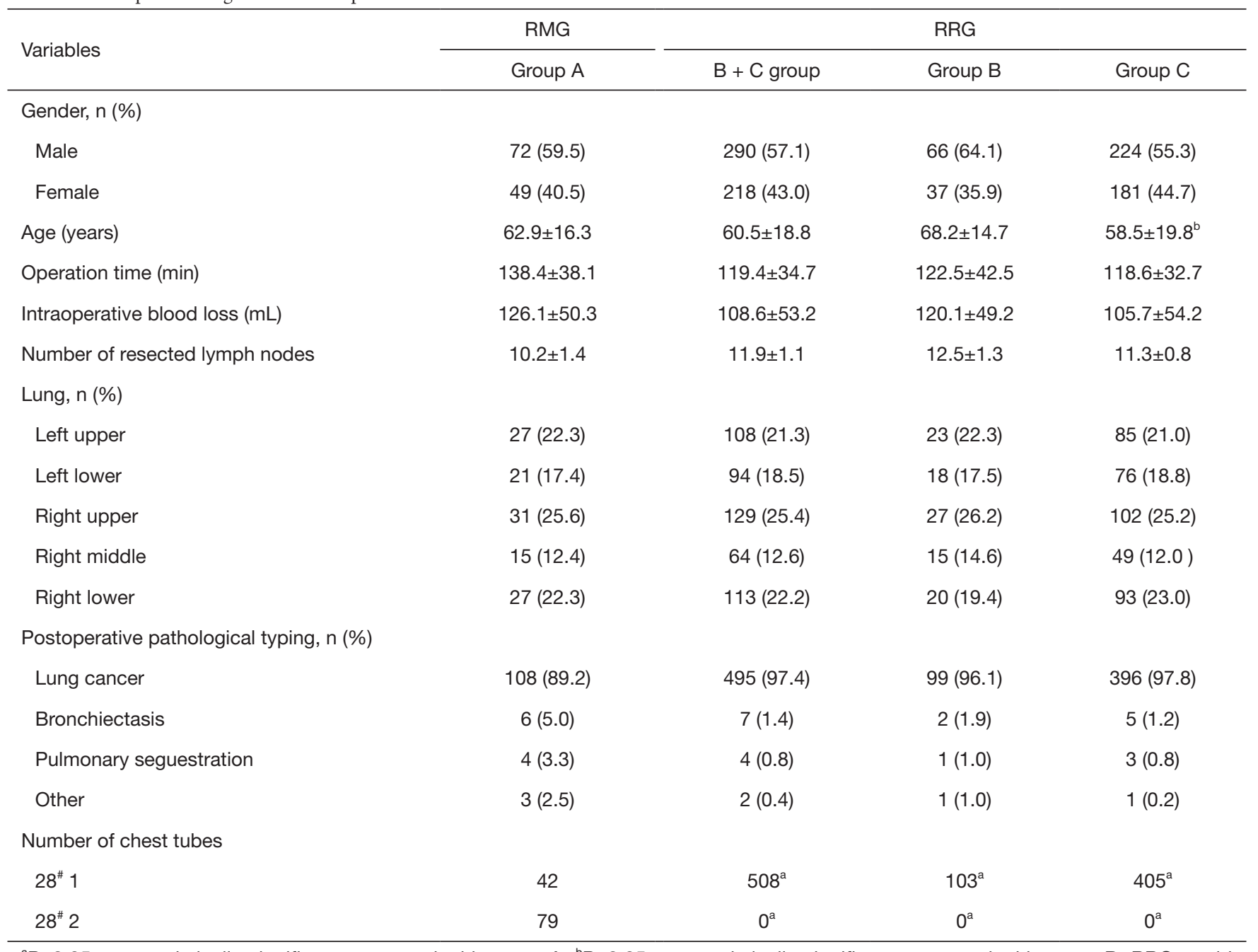

${ }^{a} \mathrm{P}<0.05$ was statistically significant compared with group $\mathrm{A} ;{ }^{b} \mathrm{P}<0.05$ was statistically significant compared with group $\mathrm{B}$. $\mathrm{RRG}$, rapid recovery group; RMG, routine management group.

accounting for $31.7 \%$. There was no significant difference between the RRG and the RMG (31.7\% vs. 29.8\%). In the RRG, 73 patients $(70.9 \%)$ had preoperative complications in group $\mathrm{B}$, and 88 patients $(21.7 \%)$ had preoperative complications in group C. Comparing group B and group A (29.8\% vs. $70.9 \%, \mathrm{P}<0.05)$. Comparing group $\mathrm{B}$ and group C $(21.7 \%$ vs. $70.9 \%)$, the $\mathrm{P}$ value was also less than 0.05 , showing a statistically significant difference. Comparing group C and group A (29.8\% vs. $21.7 \%, \mathrm{P}>0.05)$, without any statistical change. Because some patients have multiple complications at the same time, the following statistics were calculated separately (Table 4).

Compared with the RMG and RRG, the postoperative LOS, postoperative CTIT, total number of steps walked after the operation, total number of steps walked during the second $24 \mathrm{~h}$, the POD in the first 3 days after surgery, the incidence $\mathrm{PC}$, and the TC showed statistically significant improvement $(\mathrm{P}<0.05)$. In terms of postoperative LOS, the RRG $(2.74 \pm 0.80$ days $)$ had a statistically significant reduction compared with the RMG $(5.70 \pm 1.10$ days $)$; compared with group C (2.10 \pm 0.68 days) and group A, group C and group B (5.25 \pm 1.28 days) had statistically significantly shorter LOS $(\mathrm{P}<0.05)$. There was no significant difference between group $A$ and group $B(P>0.05)$. In terms of postoperative CTIT, the RMG (123.6 $\pm 34.8 \mathrm{~h}$ ) was significantly longer than the RRG $(46.1 \pm 18.5 \mathrm{~h}$ ), and the $\mathrm{P}<0.05$, showing a statistical change. group $\mathrm{C}$ was significantly shorter than group $\mathrm{A}$ and group $\mathrm{B}$. In terms of the number of steps walked on the postoperative 
Table 4 Classification of preoperative complications in the RRG

\begin{tabular}{|c|c|c|c|c|}
\hline Variables & $\frac{\text { RMG }}{\text { Group A }}$ & \multicolumn{3}{|c|}{$\mathrm{RRG}$} \\
\hline Lung infection, n (\%) & $2(1.7)$ & $11(2.2)$ & $3(2.9)$ & $8(2.0)$ \\
\hline Low lung function, $\mathrm{n}(\%)$ & $27(22.3)$ & $122(24.0)$ & $71(69.0)^{\mathrm{ac}}$ & $51(12.6)$ \\
\hline Renal insufficiency, n (\%) & 0 & $11(2.2)$ & $5(2.9)$ & $8(2.0)$ \\
\hline Three months history of organ surgery, $\mathrm{n}(\%)$ & $1(0.8)$ & $27(5.3)$ & $6(5.9)^{\mathrm{a}}$ & $21(5.2)^{a}$ \\
\hline Fundamental arrhythmia, n (\%) & $2(1.6)$ & $13(2.6)$ & $4(3.9)$ & $9(2.2)$ \\
\hline Diabetes, n (\%) & $21(17.4)$ & $92(18.1)$ & $28(27.2)^{\mathrm{a}, \mathrm{c}}$ & $64(15.8)$ \\
\hline After heart stents implantation, $\mathrm{n}(\%)$ & $1(0.8)$ & $8(1.6)$ & $6(5.8)^{a, c}$ & $2(0.5)$ \\
\hline
\end{tabular}

${ }^{a} \mathrm{P}<0.05$ was statistically significant compared with group $A ;{ }^{c} \mathrm{P}<0.05$ was statistically significant compared with group $C$. RRG, rapid recovery group; RMG, routine management group; $\mathrm{CHD}$, coronary heart disease.

day, the RMG did not get out of bed. The average number of steps walked in the RRG was 510, and the $\mathrm{P}$ value of the 2 groups was $<0.05$. There was no significant difference between groups B and C. The number of steps walked on the first day after the operation in the RRG $(4,193 \pm 2,138)$ was significantly higher than that in the RMG $(1,221 \pm 242)$. There was no significant difference between groups B and C $(\mathrm{P}>0.05)$. In terms of POD in the first 3 days after surgery, the dosage in the RRG was significantly reduced compared with that in the RMG. Meanwhile, the opioid dosage in group $\mathrm{B}$ and group $\mathrm{C}$ increased slightly, but the $\mathrm{P}$ value was $>0.05$. In terms of the proportion of PC, compared with RMG, the RRG showed a significant improvement (38.8\% vs. $18.9 \%, \mathrm{P}<0.05)$. Compared with group A (38.8\%), group C (14.6\%) showed a significant decrease. The comparison between group C and group B (14.6\% vs. 36.0\%) was also significantly reduced $(\mathrm{P}<0.05)$. There was no statistically significant change between group $\mathrm{B}$ and group A $(36.0 \% v$ s. $38.8 \%)$. In terms of total hospitalization cost after surgery, the RRG $(51,517 \pm 7,217)$ was significantly less than the RMG $(65,781 \pm 8,200)$. The cost for group $C$ was significantly lower than that of group A and group B, and there was no difference between groups B and A (Table 5 and Figure 2,3).

The incidence of $\mathrm{PC}$ was $38.8 \%$ in the $\mathrm{RMG}$, and $13.6 \%$ in the RRG. The incidence of PC in the RRG was $\mathrm{P}<0.05$ compared with that of the RMG in the incidence of continuous postoperative air leakage, atelectasis, pulmonary infection, chylothorax, pulmonary embolism, and continuous vomiting. In terms of postoperative bronchospasm, new atrial fibrillation, postoperative bleeding, outpatient thoracotomy treatment, and 30 -day readjustment, there was no significant statistical difference between the 2 groups in $\mathrm{P}$ value of $>0.05$. In the postoperative continuous air leakage, group A and group C $(8.3 \%$ vs. $0 \%)$, group B and group C $(24.3 \%$ vs. $0 \%)$, group A and group B (8.3\% vs. 24.3\%) showed statistically significant changes $(\mathrm{P}<0.05)$. In terms of postoperative atelectasis, group B and group C (2.9\% vs. $0 \%)$ and group $\mathrm{C}$ and group $\mathrm{A}(0 \%$ vs. $4.9 \%)$ showed statistically significant improvement $(\mathrm{P}<0.05)$. However, there was no significant difference between group B and group A (2.9\% vs. $4.9 \%)$ at $\mathrm{P}>0.05$. In terms of postoperative pulmonary infection, $\mathrm{P}<0.05$ in group $\mathrm{B}$ and group $\mathrm{C}(6.8 \%$ vs. $0 \%)$ and group A and group $\mathrm{C}(7.4 \% v s .0 \%)$ showed statistically significant increases, while $\mathrm{P}>0.05$ in group A and group B $(7.4 \%$ vs. $6.8 \%$ ) showed no significant differences. In terms of postoperative nausea and vomiting, compared with group B (19.8\% vs. $8.7 \%)$, the $\mathrm{P}$ values of group A and group C $(19.8 \%$ vs. $5.2 \%)$ were all $<0.05$, showing statistically significant improvement. For group B and group C $(8.7 \%$ vs. $5.2 \%)$, there was no significant difference between the 2 groups $(\mathrm{P}>0.05)$. As some patients coexisted with several complications, the following statistics were calculated separately (Table 6 and Figure 4).

The incidence of postoperative pulmonary complications in the conventional management group was $29.7 \%$, of 
Table 5 Comparison of postoperative data between RMG and RRG

\begin{tabular}{|c|c|c|c|c|}
\hline Variables & $\begin{array}{c}\text { RMG } \\
\text { Group A }\end{array}$ & \multicolumn{3}{|c|}{$\mathrm{RRG}$} \\
\hline Postoperative length of stay (d) & $5.70 \pm 1.10$ & $2.74 \pm 0.80^{\mathrm{a}}$ & $5.25 \pm 1.28$ & $2.10 \pm 0.68^{\mathrm{a}, \mathrm{b}}$ \\
\hline Chest tube indwelling time $(\mathrm{h})$ & $123.6 \pm 34.8$ & $46.1 \pm 18.5^{\mathrm{a}}$ & $100.3 \pm 51.2^{a}$ & $32.3 \pm 10.2^{a, b}$ \\
\hline \multicolumn{5}{|l|}{ Number of steps walked (steps) } \\
\hline First day after surgery & $1,221 \pm 242$ & $4,193 \pm 2,138^{a}$ & $4,083 \pm 1,957^{\mathrm{a}}$ & $4,221 \pm 2,184^{a}$ \\
\hline Opioid dose in the first 3 days after surgery $(\mathrm{mg})$ & $82.35 \pm 48.21$ & $13.09 \pm 4.82^{\mathrm{a}}$ & $15.25 \pm 5.27^{\mathrm{a}}$ & $12.54 \pm 4.71^{\mathrm{a}}$ \\
\hline Number of complications (\%) & $47(38.8)$ & $69(13.6)^{\mathrm{a}}$ & $37(36.0)$ & $32(7.9)^{\mathrm{a}, \mathrm{b}}$ \\
\hline Total cost of hospitalization (yuan) & $65,781 \pm 8,200$ & $51,517 \pm 7,217^{\mathrm{a}}$ & $63,834 \pm 6,984$ & $48,385 \pm 7,276^{a, b}$ \\
\hline
\end{tabular}

${ }^{\mathrm{a}} \mathrm{P}<0.05$ was compared with group $\mathrm{A}$ has significantly difference; ${ }^{\mathrm{b}} \mathrm{P}<0.05$ was compared with group $\mathrm{B}$ has significantly difference. RMG, routine management group; RRG, rapid recovery group.
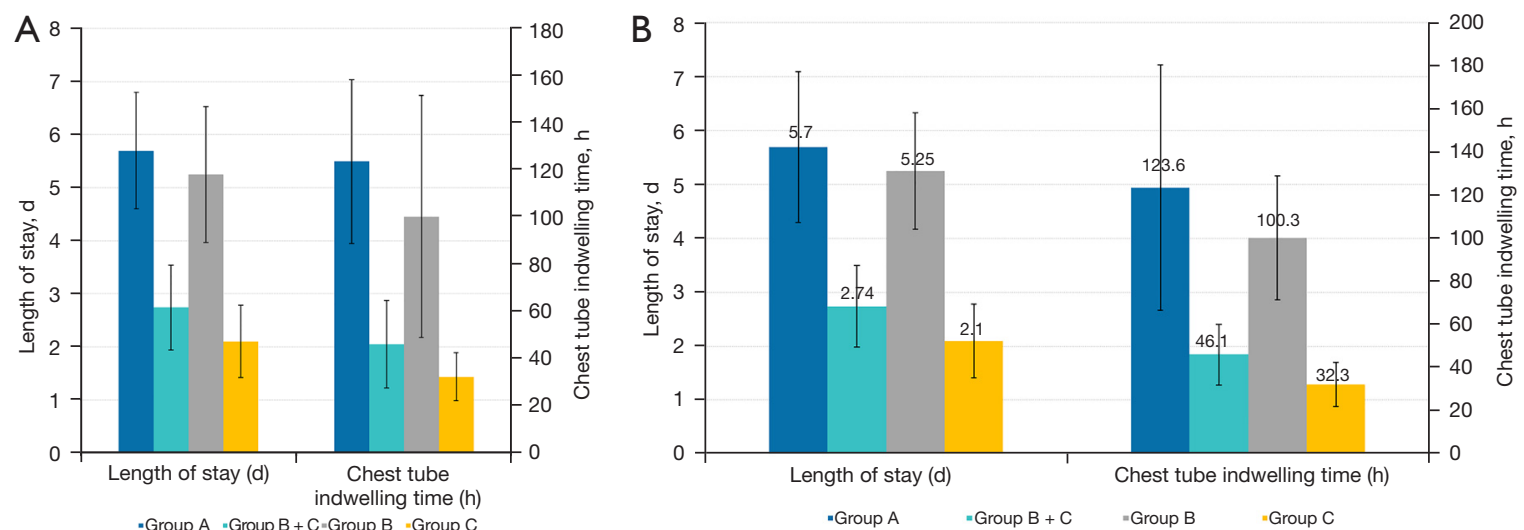

Figure 2 Comparison of postoperative LOS and chest tube indwelling time between RMG and RRG. LOS, length of stay; RMG, routine management group; RRG, rapid recovery group.

which $8.3 \%$ was sustained air leakage. The incidence of postoperative pulmonary complications was $8.5 \%$ in the RRG, which was significantly lower than that in the RMG. The incidence of postoperative pulmonary complications in group B was $37.8 \%$, of which $24.3 \%$ was sustained air leakage, and the incidence of severe pulmonary complications after the elimination of sustained air leakage was $13.6 \%$. The incidence of severe pulmonary complications in group B was lower than that in group $\mathrm{A}(\mathrm{P}<0.05)$.

The use of intraoperative anesthetic drugs was roughly similar in the RMG and RRG; however, compared to the intercostals nerve block in the RMG, closed chest line of intercostals nerve block RRG 3 days before the pain score was lower than those of group A, 3 days before the pain score $\mathrm{P}$ value $<0.05$, indicating a statistically significant improvement. At the same time, the $\mathrm{P}$ value of pain score in the first 3 days in group Bs and $\mathrm{C}$ was $>0.05$, with no significant statistical difference (Table 7 and Figure 5).

\section{Discussion}

The application of ERAS protocols to improve patients' postoperative recovery ability has been well recognized in colorectal surgery $(24,25)$. In thoracic surgery, open lobectomy can benefit from the ERAS approach, while for minimally invasive surgery, due to the reduced surgical trauma itself, it seems to be debatable whether these patients can achieve rapid recovery and less complications with the intervention of ERAS (26). Especially in recent years with the progress of minimally invasive technology, 


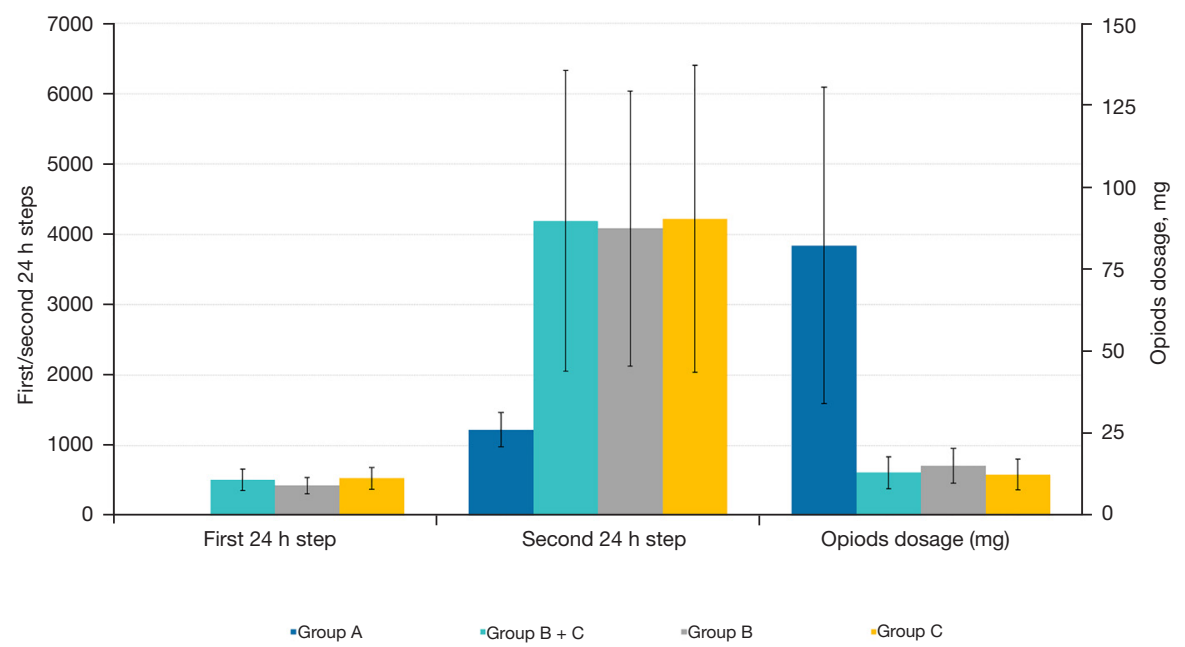

Figure 3 Comparison of postoperative steps and 3 days opioid doses between RMG and RRG. RMG, routine management group; RRG, rapid recovery group.

Table 6 Comparison of postoperative complications between the RMG and the RRG

\begin{tabular}{|c|c|c|c|c|}
\hline \multirow{2}{*}{ Postoperative complication } & \multirow{2}{*}{$\frac{\text { RMG }}{\text { Group A }}$} & \multicolumn{3}{|c|}{$\mathrm{RRG}$} \\
\hline & & Group B + C & Group B & Group C \\
\hline \multicolumn{5}{|l|}{ Pulmonary complication, $\mathrm{n}(\%)$} \\
\hline Continuous leakage & $10(8.3)$ & $25(4.9)^{\mathrm{a}}$ & $25(24.3)^{a}$ & $0^{a, b}$ \\
\hline Atelectasis & $6(4.9)$ & $3(0.6)^{a}$ & $3(2.9)$ & $0^{a, b}$ \\
\hline Acute lung injury & $3(2.5)$ & $1(0.2)$ & $1(1.0)$ & 0 \\
\hline Pulmonary embolism & $1(0.8)$ & 0 & 0 & 0 \\
\hline Asthma & $7(5.8)$ & $7(1.4)$ & $3(2.9)$ & $4(1.0)$ \\
\hline \multicolumn{5}{|l|}{ Alimentary tract complications, $\mathrm{n}(\%)$} \\
\hline New atrial fibrillation & $9(7.4)$ & $10(2.0)$ & $4(3.9)$ & $6(1.5)$ \\
\hline Myocardial infarction & $1(0.8)$ & 0 & 0 & 0 \\
\hline Postoperative bleeding, n (\%) & $3(2.5)$ & $4(0.8)$ & $4(3.9)$ & $0^{\mathrm{b}}$ \\
\hline Thoracentesis in clinic, $\mathrm{n}(\%)$ & $1(0.8)$ & $4(0.8)$ & $1(1.0)$ & $3(0.7)$ \\
\hline Pneumothorax & 0 & 1 & 0 & 1 \\
\hline Pleural effusion & 1 & 3 & 1 & 2 \\
\hline Readmission 30 days after surgery, $\mathrm{n}(\%)$ & $1(0.8)$ & $1(0.2)$ & 0 & $1(0.25)$ \\
\hline
\end{tabular}

${ }^{a} \mathrm{P}<0.05$ was statistically significant compared with group $A ;{ }^{b} \mathrm{P}<0.05$ was statistically significant compared with group. RMG, routine management group; RRG, rapid recovery group. 

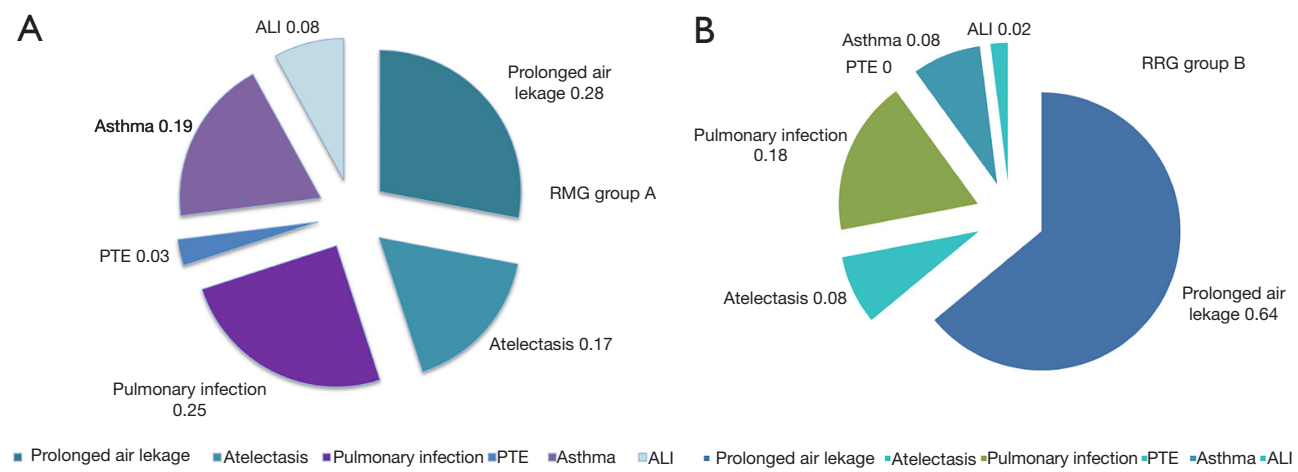

Figure 4 Comparison of postoperative pulmonary complications between group A and group B. ALI, acute lung injury; PTE, pulmonary thrombosis embolism; RMG, routine management group; RRG, rapid recovery group.

Table 7 Comparison of postoperative pain scores between the RMG and the RRG

\begin{tabular}{lcccc}
\hline \multirow{2}{*}{ Time } & RMG & & \multicolumn{2}{c}{ RRG } \\
\cline { 2 - 4 } \cline { 4 - 5 } & Group A & Group B + C & Group B & Group C \\
\hline The first day & $4.4 \pm 1.5$ & $2.3 \pm 1.1^{\mathrm{a}}$ & $2.5 \pm 1.2^{\mathrm{a}}$ & $2.2 \pm 1.1^{\mathrm{a}}$ \\
The second day & $3.9 \pm 1.3$ & $2.1 \pm 0.8^{\mathrm{a}}$ & $2.3 \pm 0.9^{\mathrm{a}}$ & $2.1 \pm 0.8^{\mathrm{a}}$ \\
The third day & $3.6 \pm 0.9$ & $1.9 \pm 0.5^{\mathrm{a}}$ & $2.1 \pm 0.7^{\mathrm{a}}$ & $1.9 \pm 0.4^{\mathrm{a}}$ \\
\hline
\end{tabular}

${ }^{a} \mathrm{P}<0.05$ was statistically significant difference compared with group $\mathrm{A}$. RMG, routine management group; RRG, rapid recovery group.

thoracoscopic surgery can significantly shorten the operation time, to achieve the results of reducing the incidence of PC and shortening the LOS. This result is consistent with the purpose of rapid recovery, so some thoracic surgeons believe that thoracoscopy is the greatest rapid recovery measure, and other rapid recovery measures need not be added to minimally invasive patients. Although the Chinese Surgery Society and Chinese Society of Anesthesiology of Chinese medical association established a consensus and guidelines in 2018 to accelerate postoperative recovery, the main focus was in the field of general surgery colorectal surgery, stomach, liver, and pancreatic duodenal surgery, and there is no guide file and consensus on thoracic surgery, Bajsová et al. shows that the ERAS protocol leads to a decrease in complications up to $40 \%$ and a reduction in hospitalization by up to $30 \%$, thereby reducing overall costs without increasing the number of rehospitalizations in gynecologic oncology (27). Also, across different hospitals, or among different doctors of the same hospital, there are varied levels of conformity to the concept of rapid rehabilitation measures, wherein some are not recognized or implemented (28).

The question has been asked whether ERAS protocols are valuable in perioperative management of minimally invasive lobectomy. In this study, the same unit managed the RMG and RRG of 2 groups according to RMG and RRG, their data were retrospectively analyzed, and it could be seen that optimization of the ERAS of process can benefit the recovery of video-assisted thoracic surgery (VATS) lobectomy patients. Such patients benefited well through adequate postoperative pain relief, which facilitated a significant increase in early postoperative activity, and reduced opioid usage allowed reduced CTIT. Concurrently, the rates PC declined, LOS was shortened, and TC was reduced. In the RRG, about $80 \%$ of patients had their postoperative LOS shortened to 3 days, which was a better result than that of Freeman $e t$ al. and Konstantinidis et al.'s research (29,30). Such results were obviously difficult to achieve in the RMG; however, even some elderly patients with extra complications can benefit from RRG implementation within $72 \mathrm{~h}$, resulting in early hospital discharge, which can greatly conserve the medical resources $(29,30)$. So the patients who underwent RRG could not achieve early hospital discharge, analysis of these patients revealed that they were more likely to be high-risk patients, with preoperative complications such as 


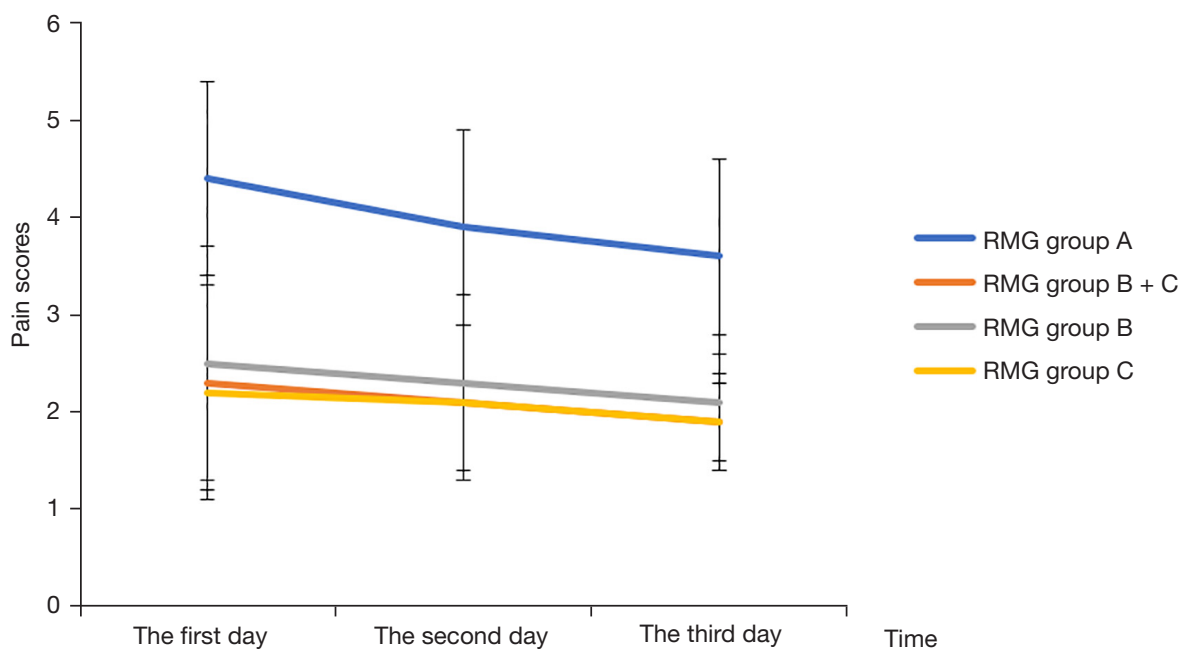

Figure 5 Comparison of postoperative pain scores between the RMG and the RRG. RMG, routine management group; RRG, rapid recovery group.

low pulmonary function, immune dysfunction after bone marrow transplantation, heart cerebrovascular stenosis, such as heart stent (3-6 months) after the recent requirement for strict anticoagulation, severe diabetes, renal insufficiency, preoperative pulmonary infection, or chest infection. Such patients need longer postoperative observation time or anti-inflammatory therapy. Some other patients could not be discharged early due to the occurrence of PC such as prolonged air leakage in the thoracic cavity, chylothorax, pulmonary infection, and new atrial fibrillation. For these patients, it is not necessary to place too much emphasis on shortening the LOS, and the treatment strategy should focus on the prevention and treatment of complications, and targeted measures should be taken for patients with different risk factors. The research showed that high-risk patients who were unable to accomplish early discharge from the hospital did not have a significantly different LOS compared to the RMG, but still experienced a reduction in level II and above pulmonary complications, therefore continuous refinement and optimization of targeted measures can help patients and ordinary high-risk patients benefit from the management of the ERAS, and it should therefore be clinically recommended.

By analyzing the reasons for refusal of ERAS management after VATS surgery, we discovered that refusal was mainly by patients who had been readmitted early after discharge, which had raised concerns regarding their immediate and long-term survival, although such concern has been shown to be redundant in colorectal surgery (5).
In other cases such as pancreatic surgery and breast surgery, the rate of readmission after ERAS has not been confirmed; in this study, the rate of readmission within 30 days for RRG participants was $0.2 \%$, which is lower than that reported by Bhagat et al., and compared with the RMG, the readmission rate had no statistically significance rise, which leads us to believe that adopting ERAS protocols in patients with VATS lobectomy is safe $(10-12,31)$. At the same time, we also found that for those who did not accept the concept of ERAS, the specific manifestations were laxness in the treatment of air leakage during the operation and hemostasis on the wound surface of lymph node bed caused by not pursuing the early removal of chest tube after the operation. Excessive dependence on the analgesic effect of systemic analgesia (intravenous pump) and opioid drugs leads to the decrease of the patient's ability to cough and expectorate phlegm and the increase of gastrointestinal reactions such as nausea and vomiting after the corresponding respiratory depression. The patient's compliance decreases with the delay of oral feeding. Early inactivity is discouraged, leading to incomplete lung dilation and increased use of anticoagulants. Therefore, it is necessary to strengthen the guidance and advertisement of ERAS guidelines after pulmonary surgery.

The European Thoracic Society developed guidelines for ERAS after pulmonary surgery in 2018, and we have also learned from clinical practice that a more optimized and rational approach design is key to patients benefiting from ERAS management (15). In our study, we adopted 
some measures that have been widely recognized, such as carbohydrate fluid prepared $2 \mathrm{~h}$ before surgery, intraoperative heating to maintain the patient's body temperature, reduce the risk of postoperative infection, and promote postoperative recovery (32). It is necessary to control intraoperative and postoperative fluid supplementation to reduce the incidence of pulmonary edema and reduce the incidence of acute lung injury after thoracic surgery (33-36). More focus should be given to detailed preoperative education, no indwelling catheterization, and postoperative reduction of thoracic drainage tube to one, and Shen et al. analysis all of the articles about enhanced recovery after lobectomy surgery that were published from October 2000 to February 2020 show that avoiding chest drain and urinary catheter placement after the surgery appears to be safe and beneficial for patients (37). At the same time, the surgeons have also accumulated some experience according to their own long-term clinical practice of minimally invasive lung resection in patients, and on this basis may make some informed adjustments to improve ERAS protocols, such as adjusting measures of postoperative analgesia, minimize the opioids dosage, emphasis on early postoperative activities, according to the preoperative complications of older age, lower lung function, diabetes, renal failure, susceptibility to infection, tendency to cardiovascular events after operation, postoperative thromboembolic high-risk patients to develop targeted countermeasures, and so on. Among these adjustment measures, we believe that intraoperative single intercostal nerve block, early postoperative removal of chest tube (preferably within the first $24 \mathrm{~h}$ ), perioperative use of COX-2 selective inhibitors, and emphasis on early postoperative activities are important improvement measures.

Intraoperative single intercostal nerve block has the advantages of simple and accurate operation, that is, it does not increase the operation time or the difficulty of the operation; however, its disadvantages are also obvious, such as the truncated analgesic maintenance time, so it needs to be coordinated with the early removal of the chest tube. Although epidural analgesia in open surgery is the gold standard in the ERAS, the incision site pain is greatly reduced with minimally invasive surgery, postoperative pain score significantly lower on the first 2 days, so we used single intercostal nerve block and intravenous NSAIDs to achieve the equivalent pain management for the first $24 \mathrm{~h}$ after surgery. If the chest tube can be pulled out separately, the use of NSAIDs can better maintain analgesia $(38,39)$. Pain scores and the opioids dosages were increased in those who could not have the chest tube removed, but overall the dose of opioids used was not large enough to be clinically significant.

In this study, the indications for chest tube removal followed by the RMG were that the amount of pleural fluid was less than $200 \mathrm{~mL} / \mathrm{d}$, and most patients needed 3-4 days after the surgery before they could meet this standard. Prospective studies have found that patients with less than $500 \mathrm{~mL} / \mathrm{d}$ of pleural fluid can have their chest tube removed after thoracoscopic surgery, which has been recommended by the guidelines developed by the European Thoracic Society (15). We pulled out the chest tube if the same indications were met, no leakage and obvious errhysis, chest drainage fluids without hemorrhage, purulent, or chylous fluid, and amount was less than $500 \mathrm{~mL} / \mathrm{d}$. According to this, about $30 \%$ of patients within the first $24 \mathrm{~h}$ after surgery were able to have their chest tube removed, and the time to chest tube removal was greatly reduced. In our study, in addition to individual patients in outpatient care: although this did not happen in the majority of patients, a pleural puncture pumping liquid, or early removal of chest tube-related to massive pleural effusion caused by repeated fever, atelectasis, and need for thoracentesis or catheter, also did not increase the occurrence of postoperative complications such as pleural infection and the probability of readmission, which showed that early removal of the chest tube is safe. However, the benefits of early removal of chest tube are that the pain score of patients significantly decreases, most patients can achieve the target analgesic effect with NSAIDs alone, and at the same time, the activity level of patients getting out of bed significantly increases, and the compliance with discharge doctor's orders also significantly increases.

It is generally believed that the use of NSAIDs can reduce the demand for opioid drugs by more than $30 \%$. The antiinflammatory analgesic effect of COX-2 selective inhibitors is better than that of general NSAIDs, and they are not associated with stomach ulcers or bleeding adverse reactions. Compared with COX-1 selective inhibitors, COX-2 selective inhibitors are currently recommended by the American Society of Anesthesiologists for perioperative preventive analgesia and multimodal analgesia drug use $(40,41)$. In our study, general use began from 2 days preoperatively to after admission, to achieve the goal of advanced analgesia, and access to postoperative intravenous preparations was maintained during the postoperative week, including until the third postoperative. Beginning on the fourth day after surgery and preoperatively in oral preparations, the study 
found that combined use intraoperative single intercostals nerve block and early removal of chest tube had a positive curative effect: the RRG group's opioids dosage was significantly reduced, these patients were able to eat earlier and more food through the mouth.

Many centers have realized the importance of early postoperative activity on the management of rapid recovery after lung resection. Khandhar et al. found that early postoperative ambulation is the key measure to reducing complications and achieving early discharge from the hospital for thoracoscopic lung resection patients (42). In their study, patients were asked to walk out of bed immediately after the endotracheal intubation was removed (42). In this study, we quantified the standard of early postoperative activity, that is, the patients were required to get out of bed after waking up and returning to the ward on the day after the surgery, and the patients were required to get out of bed with the chest tube in place at least 2 times on the first day after the surgery, each time walking for at least $6 \mathrm{~min} / 200 \mathrm{~m}$, and the total amount of walking was at least 2,000 steps within $24 \mathrm{~h}$. To ensure the implementation of this, we increased the importance of early activity in the preoperative education and operation method of introduction, intraoperative line was not inserted or the postoperative urethral catheter was removed before awakening to force patients out of the bed, the toilet ward corridor walls were hung with advertising slogans and graphics to encourage early activity. To assist the counting of steps, lengths were marked on the floor. At the same time effective analgesic measures were taken facilitate walking the length without leaking and early analgesia was given after pulling up the chest tube, which increased patients' walking compliance out of their postoperative bed. In this study, the total amount of walking of the patients in the RRG on the first day after the surgery reached an average of 4,000-5,000 m. Early activity can help the patients approach the postoperative recovery with a more positive mental state. Meanwhile, the increase of lung capacity, recovery of intestinal function, and acceleration of blood circulation are beneficial to the postoperative recovery.

By intraoperative single intercostals nerve block, early postoperative (preferably within the first $24 \mathrm{~h}$ ) removal of chest tube, perioperative use COX-2 selective inhibitors and emphasis on early postoperative activities all 4 measures of joint application, can alleviate minimally invasive lobectomy patients with early postoperative pain, decrease the dosage of opioid drugs at the same time, increase adherence of early ambulation, reduce gastrointestinal response, early postoperative activity increased obviously, along with other measures for rapid recovery, patients significantly lower incidence of postoperative complications, postoperative hospitalization time shortened obviously, therefore, these measures have clinical popularization value.

There were many shortcomings in our study. On the one hand, this was a single-center study with few patients and lack of regulation of various potential selection bias and information bias during experimental implementation. It would be beneficial to conduct a multicenter study of perioperative ERAS management regulations of thoracic surgery. On the other hand, the study lacked a rigorous pairing design, so it lacked some persuasion in analyzing the risk factors affecting the early discharge of the rapid rehabilitation group; and in the study of the cohort, the lack of rigorous rehabilitation design may have affected some of the study results, but the advantages of the rapid rehabilitation management group are self-evident.

\section{Conclusions}

In patients with minimally invasive lobectomy for chest surgery, perioperative rapid recovery management can reduce the incidence of $\mathrm{PC}$, shorten the LOS, and reduce the TC of hospitalization. Most of the patients with minimally invasive lobectomy who implemented rapid perioperative rehabilitation management were able to be discharged early within $72 \mathrm{~h}$ after operation, and the conditions were safe and reliable. Some high-risk patients cannot be discharged within $72 \mathrm{~h}$, but the incidence of grade II postoperative lung complications can be decreased and they can benefit from ERAS management. A single intercostal nerve block during the operation, removal of the chest tube as early as possible (preferably within the first $24 \mathrm{~h}$ ), and the use of a perioperative COX-2 selective inhibitor should be combined to optimize ERAS for chest surgery. Patients with lobectomy are more likely to achieve successful early discharge and rapid recovery. Therefore, it is not difficult to conclude that the ERAS can significantly reduce the incidence of postoperative complications and shorten the total length of hospital stay, bringing more benefits to patients.

\section{Acknowledgments}

Funding: None.

\section{Footnote}

Reporting Checklist: The authors have completed the 
STROBE reporting checklist. Available at https://dx.doi. org/10.21037/atm-21-6493

Data Sharing Statement: Available at https://dx.doi. org/10.21037/atm-21-6493

Conflicts of Interest: All authors have completed the ICMJE uniform disclosure form (available at https://dx.doi. org/10.21037/atm-21-6493). The authors have no conflicts of interest to declare.

Ethical Statement: The authors are accountable for all aspects of the work in ensuring that questions related to the accuracy or integrity of any part of the work are appropriately investigated and resolved. All procedures performed in this study involving human participants were in accordance with the Declaration of Helsinki (as revised in 2013). Individual consent for this retrospective analysis was waived. The ethical approval was not required.

Open Access Statement: This is an Open Access article distributed in accordance with the Creative Commons Attribution-NonCommercial-NoDerivs 4.0 International License (CC BY-NC-ND 4.0), which permits the noncommercial replication and distribution of the article with the strict proviso that no changes or edits are made and the original work is properly cited (including links to both the formal publication through the relevant DOI and the license). See: https://creativecommons.org/licenses/by-nc-nd/4.0/.

\section{References}

1. Siegel RL, Miller KD, Jemal A. Cancer statistics, 2016. CA Cancer J Clin 2016;66:7-30.

2. Zheng RS, Sun KX, Zhang SW, et al. Report of cancer epidemiology in China, 2015. Zhonghua Zhong Liu Za Zhi 2019;41:19-28.

3. Chen WQ, Li H, Sun KX, et al. Report of Cancer Incidence and Mortality in China, 2014. Zhonghua Zhong Liu Za Zhi 2018;40:5-13.

4. Roviaro G, Rebuffat C, Varoli F, et al. Videoendoscopic pulmonary lobectomy for cancer. Surg Laparosc Endosc 1992;2:244-7.

5. Wilmore DW, Kehlet H. Management of patients in fast track surgery. BMJ 2001;322:473-6.

6. Beverly A, Kaye AD, Ljungqvist O, et al. Essential Elements of Multimodal Analgesia in Enhanced Recovery After Surgery (ERAS) Guidelines. Anesthesiol Clin 2017;35:e115-43.

7. D'Andrilli A, Rendina EA. Enhanced recovery after surgery (ERAS) and fast-track in video-assisted thoracic surgery (VATS) lobectomy: preoperative optimisation and care-plans. J Vis Surg 2018;4:4.

8. Sanchez-Lorente D, Navarro-Ripoll R, Guzman R, et al. Prehabilitation in thoracic surgery. J Thorac Dis 2018;10:S2593-600.

9. Ljungqvist O. ERAS--enhanced recovery after surgery: moving evidence-based perioperative care to practice. JPEN J Parenter Enteral Nutr 2014;38:559-66.

10. Rawlinson A, Kang P, Evans J, et al. A systematic review of enhanced recovery protocols in colorectal surgery. Ann R Coll Surg Engl 2011;93:583-8.

11. Jiang W, Mao Q, Xie Y, et al. Enhanced recovery after surgery (ERAS) program in elderly patients undergoing laparoscopic hepatectomy: a retrospective cohort study. Transl Cancer Res 2020;9:4563-72.

12. Arsalani-Zadeh R, ElFadl D, Yassin N, et al. Evidencebased review of enhancing postoperative recovery after breast surgery. Br J Surg 2011;98:181-96.

13. Nakazawa S, Shimizu K, Mogi A, et al. VATS segmentectomy: past, present, and future. Gen Thorac Cardiovasc Surg 2018;66:81-90.

14. Berna P, Quesnel C, Assouad J, et al. Guidelines on enhanced recovery after pulmonary lobectomy. Anaesth Crit Care Pain Med 2021;40:100791.

15. Batchelor TJP, Rasburn NJ, Abdelnour-Berchtold E, et al. Guidelines for enhanced recovery after lung surgery: recommendations of the Enhanced Recovery After Surgery $\left(\right.$ ERAS $^{\circledR}$ ) Society and the European Society of Thoracic Surgeons (ESTS). Eur J Cardiothorac Surg 2019;55:91-115.

16. Golemi I, Salazar Adum JP, Tafur A, et al. Venous thromboembolism prophylaxis using the Caprini score. Dis Mon 2019;65:249-98.

17. Soni NJ, Franco R, Velez MI, et al. Ultrasound in the diagnosis and management of pleural effusions. J Hosp Med 2015;10:811-6.

18. Ju S, Gao Y. Advances in the Study of the Effects of Videoassisted Thoracoscopic Segmentectomy on Pulmonary Function. Zhongguo Fei Ai Za Zhi 2019;22:537-40.

19. Russotto V, Sabaté S, Canet J, et al. Development of a prediction model for postoperative pneumonia: A multicentre prospective observational study. Eur J Anaesthesiol 2019;36:93-104.

20. Woods BD, Sladen RN. Perioperative considerations for the patient with asthma and bronchospasm. Br J Anaesth 
2009;103 Suppl 1:157-65.

21. Lal D, Manocha S, Ray A, et al. Comparative study of the efficacy and safety of theophylline and doxofylline in patients with bronchial asthma and chronic obstructive pulmonary disease. J Basic Clin Physiol Pharmacol 2015;26:443-51.

22. Blanco R, Parras T, McDonnell JG, et al. Serratus plane block: a novel ultrasound-guided thoracic wall nerve block. Anaesthesia 2013;68:1107-13.

23. Clavien PA, Strasberg SM. Severity grading of surgical complications. Ann Surg 2009;250:197-8.

24. Fearon KC, Ljungqvist O, Von Meyenfeldt M, et al. Enhanced recovery after surgery: a consensus review of clinical care for patients undergoing colonic resection. Clin Nutr 2005;24:466-77.

25. Kehlet H, Wilmore DW. Evidence-based surgical care and the evolution of fast-track surgery. Ann Surg 2008;248:189-98.

26. Holbek BL, Horsleben Petersen R, Kehlet H, et al. Fast-track video-assisted thoracoscopic surgery: future challenges. Scand Cardiovasc J 2016;50:78-82.

27. Bajsová S, Klát J. ERAS protocol in gynecologic oncology. Ceska Gynekol 2019;84:376-85.

28. Chen L, Chen YJ, Dong HL, et al. Accelerate rehabilitation surgical China expert consensus and path management guide (2018 edition). Chinese Journal of Practical Surgery 2018;38:1-20.

29. Freeman RK, Dilts JR, Ascioti AJ, et al. A comparison of length of stay, readmission rate, and facility reimbursement after lobectomy of the lung. Ann Thorac Surg 2013;96:1740-5; discussion 1745-6.

30. Konstantinidis K, Woodcock-Shaw J, Dinesh P, et al. Incidence and risk factors for 90-day hospital readmission following video-assisted thoracoscopic anatomical lung resection†. Eur J Cardiothorac Surg 2019;55:666-72.

31. Bhagat R, Bronsert MR, Ward AN, et al. National Analysis of Unplanned Readmissions After Thoracoscopic Versus Open Lung Cancer Resection. Ann Thorac Surg 2017;104:1782-90.

32. Wasfie TJ, Barber KR. Value of extended warming in patients undergoing elective surgery. Int Surg

Cite this article as: Ni H, Li P, Meng Z, Huang T, Shi L, Ni B. Discussion of the experience and improvement of an enhanced recovery after surgery procedure for minimally invasive lobectomy: a cohort study. Ann Transl Med 2021;9(24):1792. doi: $10.21037 /$ atm-21-6493
2015;100:105-8.

33. Alam N, Park BJ, Wilton A, et al. Incidence and risk factors for lung injury after lung cancer resection. Ann Thorac Surg 2007;84:1085-91; discussion 1091.

34. Arslantas MK, Kara HV, Tuncer BB, et al. Effect of the amount of intraoperative fluid administration on postoperative pulmonary complications following anatomic lung resections. J Thorac Cardiovasc Surg 2015;149:314-20, 321.e1.

35. Brandstrup B, Tønnesen H, Beier-Holgersen R, et al. Effects of intravenous fluid restriction on postoperative complications: comparison of two perioperative fluid regimens: a randomized assessor-blinded multicenter trial. Ann Surg 2003;238:641-8.

36. Zeldin RA, Normandin D, Landtwing D, et al. Postpneumonectomy pulmonary edema. J Thorac Cardiovasc Surg 1984;87:359-65.

37. Shen C, Che G. No drains in thoracic surgery with ERAS program. J Cardiothorac Surg 2020;15:112.

38. Elmore B, Nguyen V, Blank R, et al. Pain Management Following Thoracic Surgery. Thorac Surg Clin 2015;25:393-409.

39. Wick EC, Grant MC, Wu CL. Postoperative Multimodal Analgesia Pain Management With Nonopioid Analgesics and Techniques: A Review. JAMA Surg 2017;152:691-7.

40. Boussofara M, Mtaallah MH, Bracco D, et al. Co-analgesic effect of ketorolac after thoracic surgery. Tunis Med 2006;84:427-31.

41. Chou R, Gordon DB, de Leon-Casasola OA, et al. Management of Postoperative Pain: A Clinical Practice Guideline From the American Pain Society, the American Society of Regional Anesthesia and Pain Medicine, and the American Society of Anesthesiologists' Committee on Regional Anesthesia, Executive Committee, and Administrative Council. J Pain 2016;17:131-57.

42. Khandhar SJ, Schatz CL, Collins DT, et al. Thoracic enhanced recovery with ambulation after surgery: a 6-year experience. Eur J Cardiothorac Surg 2018;53:1192-8.

(English Language Editor: J. Jones) 